Ann. Abeille, I965, 8 (2), I47-I59.

ÉTUDE PRÉLIMINAIRE

\title{
SUR UNE MÉTHODE D'ÉLEVAGE DES BOURDONS (BOMBUS LATR.) A PARTIR DE REINES CAPTURÉES DANS LA NATURE
}

\author{
A. POUVREAU \\ aver la collaboration technique de J. LLACII \\ Station de Recherches sur l'Abeille et les Insectes sociaux \\ Bures-sur-Yvette (Seine-et-Oise)
}

SOMMAIRE

L'auteur décrit le matériel et les méthodes qu'il a mis au point pour l'élevage des Bourdons. Des observations sur le comportement des reines de Bourdons en serre complètent les recherches ainsi qu'un exposé numérique des résultats des élevages conduits par les méthodes décrites.

\section{INTRODUC'TION}

Darwin semble avoir été le premier à signaler l'importance des Bourdons dans la pollinisation du Trèfle violet, lorsqu'il écrivait, en I859: "Les Bourdons seuls visitent le Trèfle violet (Trifolium pratense), parce que les autres Apoïdes ne peuvent atteindre le nectar. En conséquence, il n'est pas douteux que la raréfaction ou l'extinction des Bourdons en Angleterre entrainerait la diminution et même la disparition du Trèfle violet. "Il déclara, un peu plus tard, qu'il avait très rarement observé des Abeilles butinant sur les fleurs de Trèfle violet, si ce n'est à travers les perforations pratiquées dans la corolle par les Bourdons à langue courte.

Depuis longtemps, les observateurs ont remarqué les affinités naturelles qui existent entre Bourdons et fleurs de Trèfle violet. L'histoire de l'Agriculture en Nouvelle-Zélande fait état d'augmentations spectaculaires dans les rendements en graines de Trèfle violet en rapport avec l'introduction de Bourdons au siècle dernier.

Annales de l'Abeille. - 1965 . 
Les investigations effectuées par PEDERSEN (I945) au cours des années I930 à 1937 ont permis de donner une idée de l'importance des Bourdons dans la pollinisation du Trètle violet. L es observations de cet auteur ont montré qu'au début de la période de floraison, les Bourdons étaient responsables de $70 \mathrm{p}$. Ioo de la pollinisation, les Abeilles de 30 p. Ioo. Cette efficacité des Bourdons s'est encore affirmée au cours de recherches du même auteur sur la pollinisation de la Luzerne (Medicago sativa) : sur 6077 fleurs visitées par les Abeilles, 98, I p. Ioo n'avaient pas subi de déclenchement (tripping), alors que pour I 223 visites, Bombus terrestris le provoquait dans 93,7 p. Ioo des cas.

Si les Bourdons présentent par rapport aux Abeilles l'inconvénient de vivre en sociétés moins nombreuses, certains caractères d'ordre morphologique et biologique en font le plus souvent des agents pollinisateurs très efficaces. L'appareil buccal des Bourdons rappelle sensiblenent celui des Abeilles. La longueur de la langue varie d'une espèce à l'autre: elle mesure 9 à I I mm. chez Bombus terrestris, I2 à $\mathrm{I}_{4} \mathrm{~mm}$. chez Bombus lapidarius, I9 à $2 \mathrm{I} \mathrm{mm}$. chez Bombus hortorum, alors qu'elle n'est que d'environ $6 \mathrm{~mm}$. chez Apis mellifica. I' 'autre part, les Bourdons travaillent plus rapidement que les Abeilles. Ils commencent à butiner très tôt le matin et ne cessent qu'après le crépuscule lors des chaudes soirées d'été. Si les intempéries constituent un obstacle aux activités de pollinisation pour la majorité des Insectes, les Bourdons ne semblent guère affectés par des conditions météorologiques défavorables au butinage.

Les Abeilles constituant dans certains pays le seul agent pollinisateur à cause des facilités d'élevage et des possibilités de transfert des ruches à proximité des plantes à féconder, certains auteurs ont estimé que des méthodes d'élevage pouvaient être applicables aux Bourdons, afin d'utiliser ces précieux auxiliaires pour la pollinisation croisée des plantes d'intérêt économique.

\section{LES MÉTHODES D'ÉLEVAGE AC'TUELLEMENT CONNUES}

C'est Hoffer (I882) qui, le premier, incita des reines de Bourdons (Bombus lapidarius) à fonder des colonies sous le contrôle de l'homme. Quelques reines construisirent des cellules, qu'elles délaissèrent peu de temps après sans avoir déposé d'œufs.

LiNDIIARD (I9I2) et SLADEX (I9I2) ont rapporté des renseignements plus détaillés sur les tentatives qu'ils ont effectuées pour résoudre le problème de la domestication des Bourdons. LiNDHARD a utilisé des cages en bois ( $20 \times 20 \times 20 \mathrm{~cm})$, divisées en deux compartiments : l'un était recouvert d'une vitre et servait de compartiment de nourrissage, l'autre contenait des matériaux (tourbe et herbes sèches) destinés à recevoir le nid. Les deux compartiments communiquaient par un trou. Les reines de Bourdons, capturées au printemps dans la nature, étaient introduites dans les cages et alimentées au moyen d'une solution sucrée à $5 \circ \mathrm{p}$. Ioo et de pollen. Lès qu'une reine avait construit un nid, elle pouvait s'envoler librement dans une grande salle oil elle avait des bouquets de fleurs à sa disposition. LiNDIIARD réussit, au moyen de cette méthode, à faire construire 7 nids par des reines appartenant aux espèces Bombus terrestris, Bombus lapidarius, Bombus distinguendus, Bombus sylvarum et Bombus agrorum. Quelques ouvrières apparurent dans les nids.

SLADEN s'intéressa au problème de l'élevage de différentes manières. Il avait aménagé des domiciles artificiels souterrains afin d'y attirer les reines de Bourdons à la recherche d'un emplacement de nidification. Les résultats furent variables, mais peu convaincants dans l'ensemble. L'humidité et l'exposition aux ennemis constituaient le principal obstacle au développement normal des colonies. Au cours de trois années, des reines de Bombus lapidarius (66 p. Ioo), Bombus latreillellus (1 2 p. 100), Bombus terrestris (6 p. 100), Bombus hortorum et Bombus sylvarum (4 p. 100) commencèrent la construction d'un nid dans ces cavités souterraines. 
Les essais de SLADEN concernant une méthode de confinement des reines de Bourdons en cage se sont avérés d'abord infructueux. l'uis l'auteur, a introduit deux fenelles fondatrices par cage. Les résultats mont rèrent alors une légère amélioration, et, dans plusieurs cages, le développement se poursuivait jusqu'à la ponte de l'une des deux reines. A ce stade, l'une des fondatrices tuait sa compagne ; la survivante délaissait le nid et le développement de lat progéniture était alors interrompu. SLADEN réussit cependant à maintenir des reines dans leur nid en leur adjoignant des ouvrières de la même espèce capturées dans la nature, mais les colonies obtenues de cette façon étaient toutes assez réduites.

Frison (1927) utilisa des cages en bois ( $12 \times 17 \times 5 \mathrm{~cm}$ ) comportant un trou a une extrémité. Dans chaque cage, un nid, constitué d'herbes et de plusieurs couches de gaze enduite de cire, était aménagé. Un petit vase rempli d'eau miellée était disposé dans un coin de la cage. Dans certains cas, les cages étaient divisées en deux compartiments. I)ans le nid, l'auteur déposait une pelote de pollen, de la taille d'un noyau de cerise et imbibée de miel. Ene " outre à miel ", formée de cire d'Abeille, était installée en face de la pelote de pollen. Les reines de Bourdons capturées dans la nature n'étaient pas relâchées immédiatement dans les cages, mais prèalablement introduites dans des aquariums contenant du papier plissé, du miel et du pollen, et recouverts d'une gaze.

Sur un total de 46 cages, Frison obtint 5 colonics avec Bombus impatiens, 2 avec Bombus auricomus, 2 avec Bombus separalus, 8 avec Bombus bimaculalus, 2 avec Bombus feridus, I avec Bombus vagans, I avec Bombus ternarius, I avec Bombus terricola et 3 avec Bonbus perplexus. I.es tentatives d'élevage avec Bombus americanorum furent suivies d'échecs.

Frison installa également des domiciles souterrains et réusit it en faire accepter $\mathbf{r}_{7}$ sur $3^{6}$, soit $47 \mathrm{p}$. I00, au cours des années I9 5 à I9 19, par des reines de Bombus americanorum, Bombus auricomus, Bombus bimaculatus, Bombus separatus et Bombus impatiens, en quête d'un emplacement de nidification.

Les cages $(23 \times$ I $5 \times$ Io $\mathrm{cm})$ utilisées par PIATH $($ I923, I9.34) étaient recouvertes d'une vitre sur laquelle s'adaptait un couvercle en bois. Iatéralement un trou permettait l'accès dans la cage. A l'opposé de l'entrée, un morceau de cire d'Abeille était déposé sur une couche de coton constituant le matériau du nid au-dessus duquel se trouvait un petit récipient (dian. $=7 \mathrm{~cm}$; haut. $=5 \mathrm{~cm}$.) percé d'un trou sur le côté. Tous les deux ou trois jours, du pollen frais était déposé sur la cire. Chaque cage contenait également un récipient d'une solution d'eau miellée, et, dans un coin, un petit tas de sable sec pour les besoins sanitaires.

Plath introduisait dans chaque cage I reine et a à 3 ouvrières capturées dans la nature. Dès que la reine et les ouvrières avaient accepté le nid artificiel et que des ceufs avaient été déposés, les Bourdons pouvaient sortir pour butiner lorsque les conditions météorologiques étaient favorables. Plath avait remarqué que, dans plusieurs cas, les reines et les ouvrières construisaient des nids à l'extérieur du petit récipient ; elles sécrétaient alors elles-mêmes la cire qui servait de base à la première cellule à ceufs. PLATI obtint ainsi plus de 20 colonies de Bourdons pendant les amnées 1921 à 1927.

JORDAN (I936) maintenait des reines de Bourdons confinées séparément dans de petites cages d'observation à moitié remplies de mousse finement dilacérée. Les cages étaient entreposées dans une cavé à une température voisine de $60 \mathrm{C}$, pendant I à i i jours, avant d'être placées en plein air où les reines pouvaient voler librement. Par cette méthode, 2 reines sur I 3 de Bombus musconum ont fondé des colonies en 1929, et 4 sur i i en 1930. Afin d'obtenir de meilleurs résultats, JoRDAN préféra utiliser des reines capturées dans la nature au moment où elles recherchaient un emplacement de nidification.

Fye et MEDLER (I954) ont adopté les méthodes de SIADEN et de Frison pour attirer les reines de Bourdons dans des " domiciles " artificiels souterrains, consistant en boîtes en bois, pots à fleurs, cônes en papier goudronné, tuiles. Ces "aménagements " étaient situés dans des fossés, des vergers et divers autres lieux, et installés à l'automne. Sur I 54 "domiciles ", 27 p. Ioo furent occupés par des fourmis, 34 p. Ioo par des reines de Bourdons (Bombus rufocinctus, Bombus separatus, Bombus borealis, Bombus fervidus, Bombus vagans), et le reste par des mulots.

Free et Butler (1959) ont mené leurs investigations sur les Bourdons à partir de 6o colonies de Bombus pratorum obtenues en cages. Deux de ces colonies furent fondées par des reines seules, les autres par des reines aidées d'ouvrières capturées dans la nature.

Les essais de domestication entrepris par VALLE (I955) étaient entièrement basés sur la méthode d'Hasselrot (I952). Iin I953, sur 130 reines de Bombus lucorum, Bombus hypnorum, Bombus agrorum, Bombus lapidarius, Bombus pratorum, Bombus equestris, Bombus hortorum et Bombus distinguendus, $57 \mathrm{p}$. Ioo construisirent des nids et, en I954, $4^{8} \mathrm{p}$. IOo. VALLE a signalé que Bombus lucorum commençait rapidement la construction d'un nid; de même que Bombus hypnorum et Bombus agrorum; Bombus lapidarius et Bombus pratorum étaient de médiocres "constructeurs". Les tentatives d'élevage effectuées avec Bombus equestris, Bombus hortorum et Bombus distinguendus se sont soldées par des échecs.

Dans leurs travaux sur le vol des Bourdons, STEIN (1956) et LehMENSICK (1956) ont utilisé des cages du modèle adopté par IINDHARD. I,es reines de Bourdons, capturées dans la nature, étaient relâchées dans une serre $(\mathrm{I} 2 \times 5 \mathrm{~m}$.) en présence de cages pour la construction des nids. 
La méthode mise au point par Hasserrot (1952, 1960) peut être ainsi décrite : les reines de Bourdons, capturées au printemps, étaient introduites, après avoir séjourné pendant quelque temps dans un réfrigérateur à une température de $-5 \grave{a}+3^{\circ} \mathrm{C}$, dans des cages $(30 \times 30 \times 18 \mathrm{~cm}$. $)$ à deux compartiments. Le compartiment de nidification était rempli de mousse aux $2 / 3$ avec, au milieu, de l'ouate cellulosique sur laquelle était déposée une boulette de pollen, de la taille d'une noisette. Le compartiment de nourrissage contenait une petite cupule d'eau miellée. Aussitôt après la ponte, le compartiment de nidification était installé en plein air.

Les résultats enregistrés par HasSELRoT, basés sur le nombre de reines saines c'est-à-dire dépourvues de parasites (Sphaerularia bombi, Nosema bombi,...) ont porté sur plusieurs années (1952, 1953, 1954, 1958) : 68 reines de Bombus terrestris sur 7.3 (93 p. 100) ont construit des nids, 5 sur 6 (83 P. 100) de Bombus lucorum, 22 sur 41 (54 p. 100) de Bombus lapidarius, 31 sur 35 (89 p. 100) de Bombus hypnorum, 9 sur 23 (39 p. 100) de Bombus agrorum, 6 sur 7 (86 p. 100) de Bombus pratorum et I sur 5 de Bombus ruderarius.

Norgana Holm (1954, 1960) a utilisé une méthode d'élevage semblable à celle d'Hasselrot. Le nombre de reines qui fondèrent des colonies fut de 12 sur $3^{\circ}\left(4^{\circ} \mathrm{p}\right.$. 100) pour Bombus terrestris, et $2 \operatorname{sur} 6$ (33 p. Ioo) pour Bombus lapidarius. Les essais entrepris avec d'autres espèces furent nuls. En 1958 , N. IIolm modifia sa technique d'élevage. Il introduisit, par un orifice latéral du compartiment de nourrissage, un tube de verre percé à son extrémité inférieure, où les reines de Bourdons pouvaient aspirer une solution sucrée à $5^{\circ} \mathrm{p}$. 100 . Les pourcentages de colonies fondées furent alors de 73 p. 100 pour Bombus lapidarius et de 50 p. 100 pour Bombus terrestris, tandis qu'en 1959, ces taux étaient respectivement de $17 \mathrm{p}$. 100 et de 27 p. 100. Les reines étaient capturées dans la nature au printemps, ou bien provenaient de serres non chauffées, destinées à l'hibernation des Insectes.

Zaptetal (1961) a utilisé des ruches $(20 \times 20 \times 25 \mathrm{~cm}$.) et des petites cages placées à l'intérieur d'une volière $(90 \times 80 \times 60 \mathrm{~cm}$.). Les ruches avaient des parois calfeutrées et étaient remplies aux $2 / 3$ de " coton de couturier " et de mousse sèche finement découpée. L'ouverture consistait en un passage ménagé entre les deux parois latérales de la ruche. La ventilation était assurée par un grillage recouvrant la ruche. Les reines ayant hiverné artificiellement étaient disposées dans une volière; les reines capturées dans la nature étaient introduites dans des cages à envol libre. Les Bourdons étaient alimentés au moyen d'une solution d'eau miellée et de pollen. Après la construction du nid, le pollen était distribué directement dans les cellules.

Les colonies étaient transportées dans les champs, à proximité des plantes à polliniser, notamment le Trèfle et la Luzerne.

Horber (t96i) a entretenu des élevages de Bombus hypnomum à partir de reines ayant hiverné en réfrigérateur à des températures de $-\mathrm{I}^{\circ} \mathrm{C} \grave{a}+1^{\circ} \mathrm{C}$.

A la fin de la période d'hibernation, les reines étaient introduites dans des cagettes en bois disposées dans une chambre à une température de $15^{\circ}$ à $20^{\circ} \mathrm{C}$, et nourries d'eau miellée et de sirop de sucre. Après un séjour d'une semaine dans ces conditions, les reines qui manifestaient une certaine activité étaient alors transférées dans une pièce à des températures variant de $25^{\circ} \mathrm{C} \dot{a} 33^{\circ} \mathrm{C}$, sous un éclairage artificiel permanent, et avec une nourriture abondante (eau miellée), en vue de la fondation de colonies.

Les cages utilisées pour l'élevage des colonies étaient inspirées d'un modèle mis au point par HASSELROT : chaque reine était disposée dans une cage à deux compartiments communiquant par un petit trou à travers la cloison. L'un des compartiments $(20 \times 20 \times 20 \mathrm{~cm}$.), complètement fermé, était destiné à recevoir le nid et ne contenait aucun matériau. L'autre compartiment, recouvert d'une vitre, constituait le vestibule contenant un nourrisseur (eau miellée). Une boulette de pollen, de la grosseur d'une noisette, était disposée dans le compartiment de nidification.

HORBER considérait essentiellement l'influence des facteurs trophiques et climatiques (température, lumière). L'apport en pollen ne s'effectuait qu'à certains stades du développement de la colonie. Le pollen était fourni sous forme de pelotes, tel qu'il était recueilli dans les trappes et déversé directement sur quelques cellules. Il n'y avait aucun approvisionnement en fleurs fraîches.

Ainsi, les méthodes d'élevage des Bourdons ont fait l'objet de plusieurs procédés d'investigation :

Io Aménagement de domiciles souterrains, ou à la surface du sol, afin d'attirer les reines de Bourdons à la recherche d'un emplacement de nidification. Ce procédé, plus primitif, est généralement abandonné, car les autres méthodes se sont avérées plus pratiques.

$2^{\circ}$ Transfert de nids fondés dans la nature. La période la plus favorable à cette opération se situe après le développement de la première ponte, afin d'éviter la dé- 
sertion de la reine. I,es nids peuvent être transférés en serre, ou en cages, à proximité de plantes dont on désire assurer la pollinisation.

$3^{\circ}$ Capture de reines dans la nature; introduction de celles-ci en serre ou en cages pour stimuler leur instinct de nidification. Ies variantes de cette méthode consistent à introduire directement les reines dans des cages, en salle d'élevage, ou bien de laisser séjourner préalablement les Bourdons en serre, avant de les inciter à choisir un emplacement de nidification.

$4^{\circ}$ Contrôle complet du cycle biologique des reines en confinant les fondatrices et les colonies dans des cages, en serre ou en salle d'élevage, où tous les facteurs (température, lumière, hygrométrie) peuvent être rigoureusement contrôlés.

Étant donné l'importance et l'extrème variabilité des facteurs écologiques et éthologiques qui caractérisent les recherches sur les Bourdons, il apparaît que des études particulières sont nécessaires pour chaque climat et chaque espèce pollinisatrice, de même que pour chaque espèce végétale ou chaque type de culture à polliniser, d'où la diversité des méthodes d'élevage et la fluctuation des résultats d'une année à l'autre.

Le but de la méthode d'élevage que j'ai mise au point est de répondre à certains problèmes physiologiques et écologiques concernant l'aptitude des reines de Bourdons à fonder des colonies, en tenant compte du fait que ces Insectes sont capturés dans la nature et, par conséquent, échappent dans une certaine mesure au contrôle des facteurs abiotiques qu'ils ont subis au cours de l'hibernation. Cette méthode ainsi mise au point ne peut constituer qu'une étape dans la voie de la domestication complète des Bourdons.

\section{EXPOSÉ DE LA MÉTHODE D'ÉLEVAGE}

\section{Conditions de capture des reines de Bourdons}

Les reines de Bourdons capturées au printemps (I964) provenaient de la région du Hurepoix, au sud-ouest de Paris, caractérisée par un relief accidenté et de nombreuses vallées aux versants recouverts d'étendues boisées. Les fonds de ces vallées sont humides et favorisent le plus souvent le développement de cultures maraîchères et fruitières.

Les reines de Bourdons sont capturées au moment où elles survolent, à basse altitude, les aires de végétation herbacée, le long des haies, sur les talus, ou en lisière des bois, pour $\mathrm{y}$ rechercher les orifices des terriers et les visiter, ou bien lorsqu'elles butinent sur les fleurs de Saules ou des autres plantes pollénifères ou nectarifères.

Les périodes de floraison des espèces végétales fréquentées par les reines de Bourdons s'étagent de la mi-mars à la fin du mois de mai. Ces espèces comprenaient principalement des saules (Salix cap̈raea, Salix viminalis, Salix cinerea), des jacinthes sauvages (Endymion nutans), des pissenlits (Taraxacum dens-leonis), des Rosacées (Cerasus avium, Prunus domestica, Pirus communis, Malus communis), des Labiées (Lamium album, Lamium galeobdolon), des Papilionacées (Trifolium pratense, Cutisus laburnum, Robinia pseudacacia, Medicago sativa, Trifolium incarnatum). 
Les reines des diverses espèces de Bourdons étudiées, les plus communes dans cette région, apparaissent à des périodes différentes du printemps; successivement : Bombus pratorum, Bombus terrestris, Bombus lucorum, Bombus hypnorum, Bombus lapidarius, Bombus agrorum.

Les femelles capturées sont introduites individuellement dans des flacons en verre et placées dans un récipient contenant de la glace, afin d'éviter qu'elles ne soient perturbées pendant le transport.

Les reines, encore engourdies, sont marquées sur le thorax d'un point de peinture de couleur différente selon leur provenance, puis libérées dans la serre; les insectes agités subissent préalablement une légère anesthésie par le gaz carbonique.

\section{Matériel utilisé}

Les Insectes sont abrités dans une petite serre $(4,00 \times 3 \times 2 \mathrm{~m})$, munie de deux fenêtres grillagées pour assurer la ventilation, et contenant des cages destinées à héberger les reines (photo $x$ ).

I,e conditionnement thermique et hygrométrique de la serre est assuré par des appareils adéquats et contrôlé quotidiennement par enregistrement; on maintient

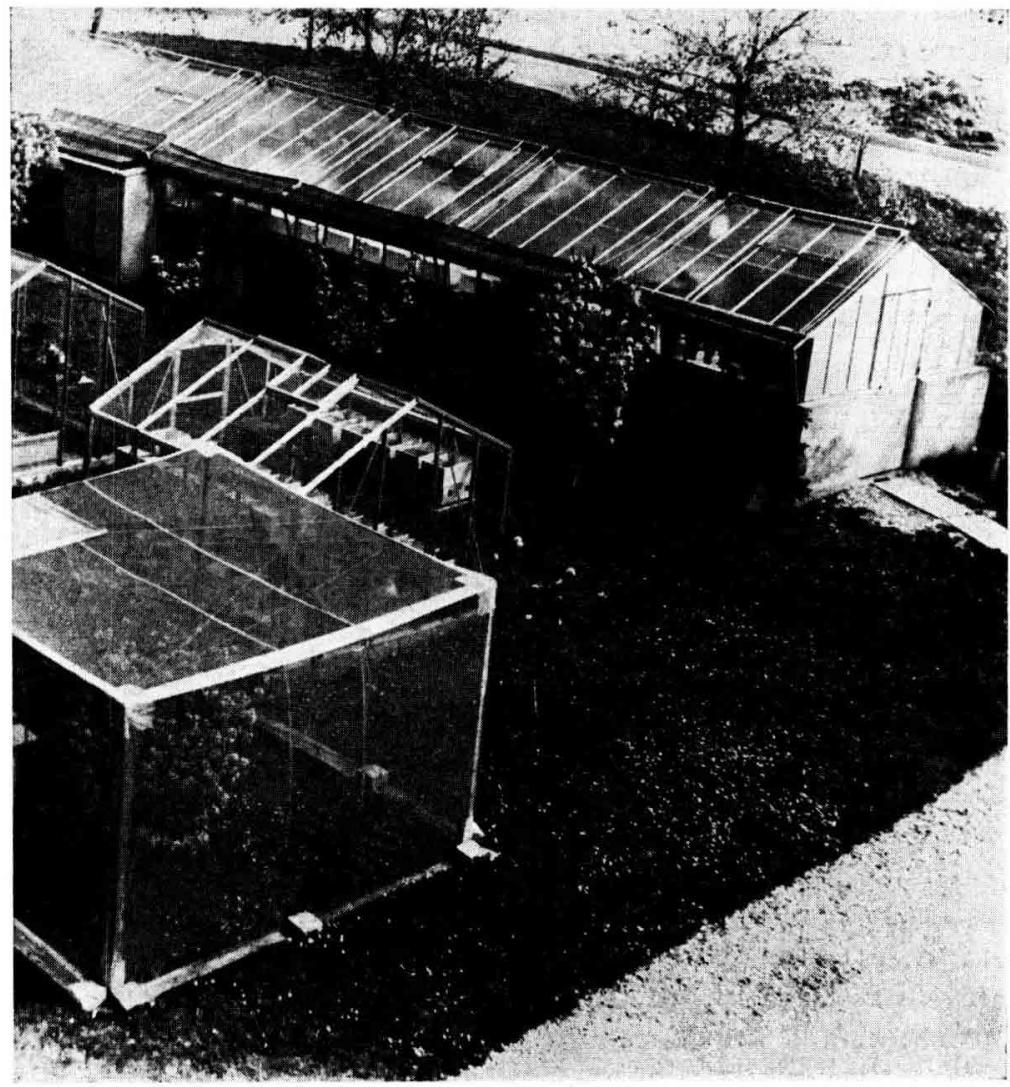

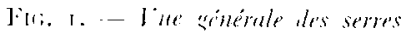


dans celle-ci des températures variant de ${ }^{\circ} 8^{\circ} \mathrm{C}$ à $27^{\circ} \mathrm{C}$ en photopériode, et de $\mathrm{I} 2^{\circ} \mathrm{C}$ à $\mathrm{I} 7^{\circ} \mathrm{C}$ en scotopériode, et un degré hygrométrique oscillant entre 60 et $75 \mathrm{p}$. Ioo. Les Insectes sont soumis au simple éclairement naturel.

Les cages, en bois, sont constituées de deux compartiments (photo 2) : un

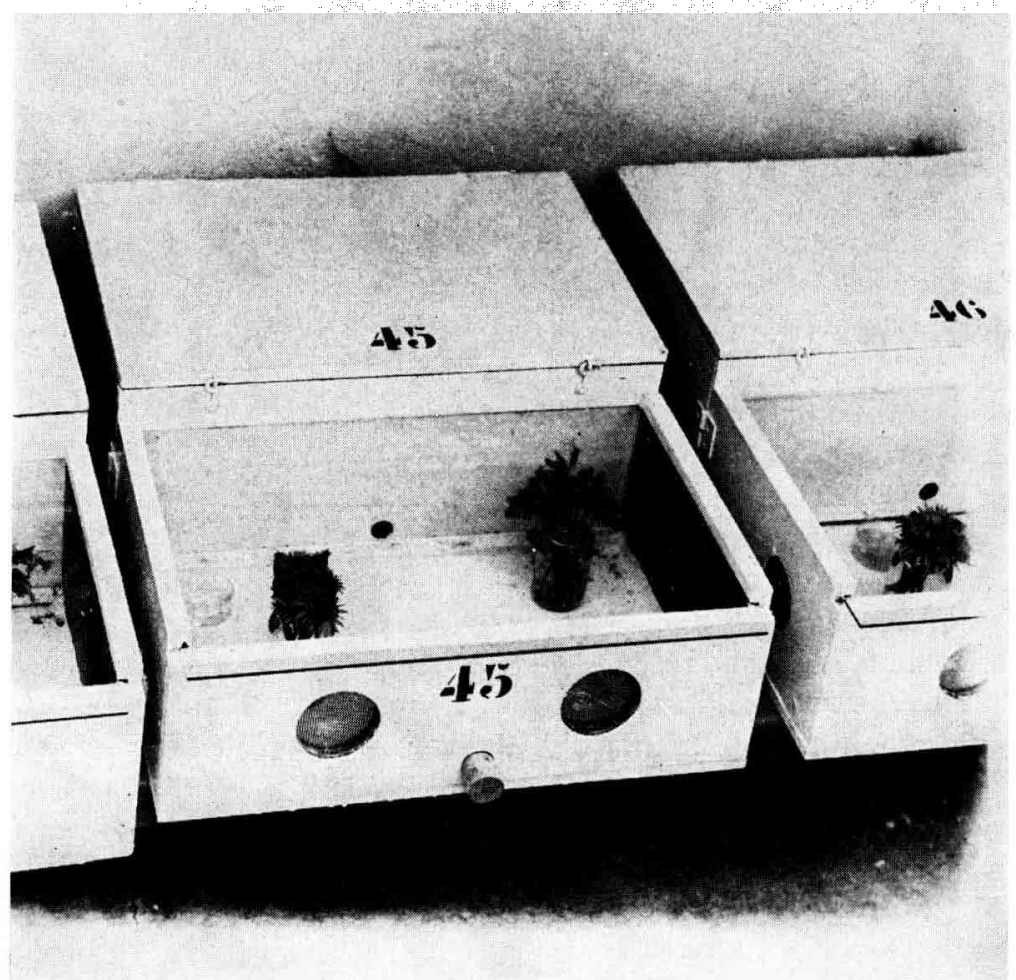

FIG. 2. - Type de cage utilisé pour l'éleange des bourdons

compartiment de nidification $(42 \times 28 \times 20 \mathrm{~cm})$, recouvert intérieurement d'un isolant thermique, la frigolite (1), afin de réduire les brusques changements de la température extérieure, et contenant divers matériaux en vue de l'édification du nid ; un compartiment de vol $(42 \times 26 \times \mathrm{I} 2 \mathrm{~cm})$, virté à la partie supérieure, pourvu de hublots grillagés pour permettre la ventilation, et contenant des récipients pour l'alimentation des Bourdons. Un orifice de $1,5 \mathrm{~cm}$ de diamètre permet la communication entre les deux compartiments, dont la fixation est assurée par un système de charnières. L es cages sont peintes en blanc afin de faciliter le repérage de l'orifice d'accès par les bourdons, et pour assurer leur entretien lors de leur utilisation en plein champ. Nous avons utilisé également un autre type de cage, à double compar-

(1) l'olystyrène vellulaire rigicle à faible jooids spécificue. 
timent, mais de dimensions différentes : compartiment de nidification : $25 \times 20 \times$ $23 \mathrm{~cm}$; compartiment de rol : $25 \times 40 \% 30 \mathrm{~cm}$.

Divers matériaux ont été mis à la disposition des Insectes : des Sphaignes, sous forme de fibres séchées provenant du Janemark, du coton cardé, préalablement dilacété, du kapok et du crin extraits de vieux matelas, de fines Graminées ainsi que des mousses desséchées.

\section{Alimentation des Insectes}

L'apport d'aliments a constitué l'une de nos préoccupations essentielles : du miel, ainsi qu'une solution sucrée à 50 p. Ioo (sucre glace - eau), étaient fournis aux Insectes dans des boîtes de Pétri remplies de billes de verre, afin d'éviter l'engluement des pattes et du corps des Bourdons. Ces solutions étaient renouvelées deux à quatre fois par semaine pour empêcher le déreloppement des moisissures et la pollution par les Bourdons. Des fleurs abondantes et variées, en rapport avec la période de floraison, étaient mises à la disposition des Insectes volant librement en serre, ou dans les cages lorsque les reines ý étaient confinées. Les Bourdons disposaient également de pollen pulvérisé ou en pelotes, provenant de trappes à pollen de ruches d'Abeilles.

\section{Procédé pour induire les reines à jonder des colonies}

Les reines de Bourdons nouvellement sorties de leur torpeur hivernale manifestent une grande activité dans la recherche de la nourriture et procèdent au choix d'un emplacement de nidification.

La suppression de la vitre amovible du compartiment de vol permet aux reines un libre accès dans les cages. La période qui précède la fondation de la colonie, et au cours de laquelle la reine recherche un emplacement pour nidifier, peut être d'une durée plus ou moins longue, et le meilleur moment pour disposer une reine en cage est difficile à déterminer. Il convient d'éviter un confinement prématuré dans les cages. Aussi, nous a-t-il semblé opportun, du moins avec des Insectes dont nous ne connaissions pas la date d'émergence, de permettre un libre accès des reines dans les cages oì elles avaient élu domicile. FRIDEx (I 964 ) estime qu'une nidification normale, ne peut avoir lieu que si les reines virent préalablement pendant une dizaine de jours en conditions naturelles.

Iès l'émergence des reines, au printemps, s'effectue la vitellogenèse. Ce processus physiologique important ne peut se produire que chez des femelles normales, c'est-àdire exemptes de parasites, en particulier Sphaerularia bombi, et aptes à recouvrer rapidement des activités de butinage; en effet, après une période plus ou moins longue d'hibernation, des conditions météorologiques défavorables peuvent entraîner un affaiblissement physiologique des reines, préjudiciables à une reprise normale de l'activité. Les exigences fondamentales des reines pour fonder des colonies, portent, d'autre part, sur des facteurs trophiques et éthologiques particuliers à chaque espèce. Mais nos connaissances sont encore limitées en ce qui concerne la valeur alimentaire du pollen des diverses espèces végétales fréquentées par les Bourdons, ainsi pue l'action de ces pollens sur la croissance orocytaire. L'éthologie des diffé- 
rentes espèces de Bourdons peut également intervenir dans l'alimentation et favoriser la maturité des gonades. Des connaissances insuffisantes des conditions exigées par les Bourdons ne nous ont pas permis d'intervenir plus directement dans le contrôle des reines clestinées à fonder des nids.

\section{OBSERVATIONS SUR LE COMPOR'TEMENT}

\section{DES REINES EN CONFINEMENT}

Le comportement des reines introduites en serre est généralement caractéristique de leur état physiologique. Après quelques vols de reconnaissance, plusieurs tendances se manifestent parmi les Insectes. Les Bourdons récemment sortis d'hibernation ont un comportement appétitif, dont les manifestations sont plus évidentes, souligné par la fréquence et l'importance de la consommation alimentaire. Ces insectes effectuent des visites prolongées sur les fleurs, séjournant parfois pendant quelques heures sur celles-ci avant de reprendre leurs activités de butinage.

L,es reines émergées plus précocement opèrent d'une façon plus fébrile, visitant de nombreuses fleurs avant de s'envoler, les corbiculae remplies de pollen, vers leur nid. W'autres reines, au comportement aberrant, témoignent le plus souvent d'une atteinte parasitaire par des Nématodes, Sphaerularia bombi (Pouvreau, I962). L,es Bourdons infestés marquent une certaine répugnance au vol, se déplaçant seulement pour s'alimenter ou fuir lorsqu'ils sont perturbés. En serre ou en cage, ces insectes ont un vol lourd, se heurtant violemment contre les parois, parfois contre les obstacles.

L'orientation des cages en serre n'a pas paru déterminante dans le choix exercé par les reines. Plus importante, semble-t-il, a été la nature des matériaux mis à la disposition des fondatrices dans les compartiments de nidification. Après les périodes d'exploration des cages et de construction des nids, nous avons pu constater la dépendance qui existait entre les matériaux de nidification et les différentes espèces de Bourdons. C'est ainsi que le coton cardé, les fibres de Sphaignes et le kapok sont les matériaux les plus recherchés par Bombus lapidarius, Bombus terrestris et Bombus hypnorum. Bombus agrorum et Bombus variabilis construisent leurs nids de préférence dans de fines herbes sèches. Tous ces matériaux, à la texture souple et dense, peuvent être travaillés par les reines et agencés en une cavité de nidification, en communication avec l'extérieur par une ou plusieurs galeries de longueur variable. Le crin végétal et les mousses desséchées, aux fibres plus rigides, moins malléables, ne présentent pas les mêmes qualités d'isolement et de "moelleux ", et sont peu employés par les Bourdons. Ces observations corroborent ce que nous savons sur la diversité des matériaux qui composent les nids de Bourdons dans la nature, malgré des connaissances encore trop fragmentaires.

I, a construction d'un nid se manifeste par quelques signes : la reine butine sur les fleurs ou récolte le pollen broyé mis à sa disposition dans un récipient, constituant rapidement des pelotes de pollen; la reine recueille en abondance miel et solution sucrée qu'elle régurgite, dès son retour au nid, dans une cupule cireuse, première étape dans l'édification du nid. Lorsqu'on intervient dans la cage, la reine 
préserve son nid en se plaçant sur les matériaux, dans une attitude caractéristique de défense, c'est-à-dire sur le dos, l'extrémité de l'abdomen tournée vers l'intrus ; une fois le danger écarté, la reine pénètre dans son nid en ramenant des matériaux, comme pour en interdire l'accès.

Nous avons effectué des expériences afin de déterminer quelle était la meilleure époque pour disposer les cages en plein air, c'est-à-dire avec le moindre risque d'abandon de la cage par la reine. Les premiers essais ont été réalisés en plaçant la cage dans une volière grillagée $(3 \times 3 \times 2,5 \mathrm{~m}$.), où l'insecte disposait de pollen et de miel en abondance. Il semble, d'après nos observations, que la reine peut être laissée en liberté dès le début de la construction du nid, et plus précisément, lorsque la cupule cireuse, destinée à recevoir la première réserve de miel, est édifiée. Dans ce cas, le retour au nid se trouve assuré par une motivation plus forte, le transport de la nourriture ; ce sont les conditions physiologiques de la reine qui sont régulatrices de son activité et l'incitent à rejoindre son nid.

\section{RÉSULTATS}

Les résultats obtenus au cours de l'année I964 sont rapportés dans le tableau I.

\section{TABITHAU I}

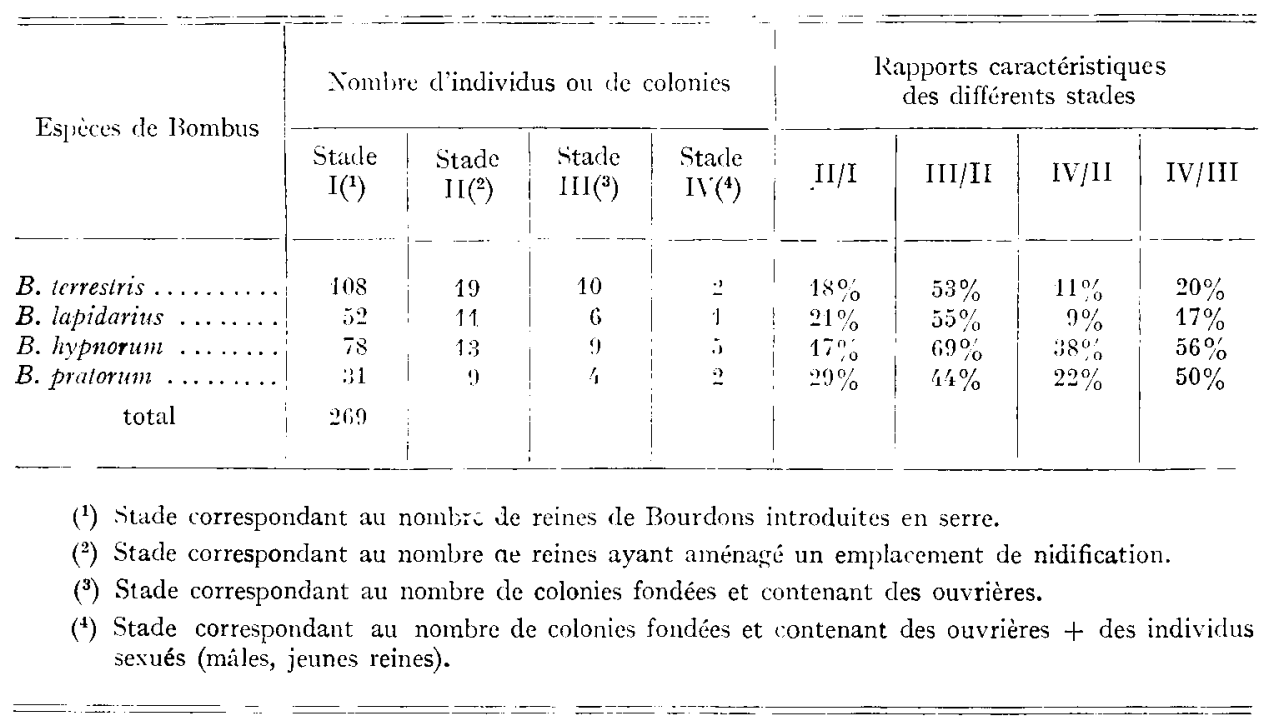

A l'examen de ce tableau, quelques remarques s'imposent, notamment en ce qui concerne les faibles pourcentages de fondations de colonies obtenus par rapport au nombre de reines capturées dans la nature. Plusieurs explications sont alors possibles : une proportion importante de fondatrices sont parasitées par Sphaerularia bombi; la présence de ces Nématodes chez les reines de Bourdons inhibe 
la croissance des ovaires, empêche la ponte et la fondation de colonies. L'intensité d'infestation des reines de Bourdons est étroitement soumise à des variations locales ou saisonnières. L,es taux de parasitisme par Sphaenularia bombi observés chez les reines de Bourdons introduites en serre étaient de 44 p. roo pour Bombus terrestris, 65 p. Ioo pour Bombus lapidarius, 46 p. Ioo pour Bombus hypnorum, et 28 p. Ioo pour Bombus pratorum.

D'autre part, le nombre de reines introduites en serre était trop élevé par rapport au nombre de cages disponibles (40), ce qui a entraîné la perte de nombreuses fondatrices par suite de leur intrusion dans des cages déjà occupées. Ėn effet, 1'agressivité des reines nidifiant envers les importuns se manifeste essentiellement au cours des premiers stades de développement de la colonie; les degrés d'agressivité sont d'ailleurs variables suivant 1'espèce envisagée, Bombus hypnorum se montrant plus agressive que Bombus terrestris et Bombus lapidarius, et la nature de l'agression, celle-ci pouvant aller de la simple expulsion hors du nid au combat entraînant la mort, le plus souvent de l'intruse.

Certaines reines sortent très affaiblies en fin d'hibernation par épuisement de leurs réserves nutritives (corpus adiposum) ; l'insuffisance de leurs ressources énergétiques, ne leur permettant pas de répondre aux intenses activités de butinage, provoque leur dépérissement. La vigueur de la reine peut être réduite par de mauvaises conditions climatiques pendant la période d'hibernation, ou la sortie prématurée de la reine au printemps. Bien que le cycle d'hibernation des Bourdons semble être étroitement lié à la température et à la phénologie des floraisons, des conditions météorologiques défavorables (refroidissement, humidité) survenant au printemps peuvent également être préjudiciables à la fondation des colonies.

\section{CONCLUSIONS}

La méthode d'élevage exposée précédemment contribue à élargir nos connaissances sur quelques-unes des conditions requises pour induire les reines de Bourdons à fonder des colonies.

Certains facteurs, notamment des facteurs trophiques, ont une influence déterminante sur les exigences fondamentales des reines pour la fondation des colonies. I,es besoins qualitatifs et quantitatifs en pollen des Bourdons sont variables selon les espèces, puisque celles-ci émergent au printemps à des périodes différentes, où se succèdent les floraisons des diverses espèces végétales. C'est par incertitude des exigences trophiques des reines qu'il nous a semblé préférable de laisser celles-ci évoluer librement en serre, abondamment pourvue de provende, plutôt que de les confiner prématurément en cages. Seul le contrôle complet des reines au cours des périodes de reproduction et d'hibernation pourrait permettre de déterminer, de manière plus précise, les besoins physiologiques des femelles fondatrices. Cette méthode aurait, de plus, 1'avantage de pouvoir procéder à l'élimination des causes d'infestations parasitaires, en particulier de Sphaerularia bombi, et de soustraire la reine aux facteurs abiotiques défavorables.

La réponse à différentes conditions de température constitue également un facteur important pour "forcer " les reines à construire un nid. Le réchauffement 
de la serre au début du printemps s'est avéré nécessaire pour favoriser la disposition des reines à rechercher un emplacement de nidification, les conditions extérieures. n'étant guère favorables.

Nos connaissances sont cependant limitées en ce qui concerne la nature exacte des stimuli qui induisent la ponte et l'élevage des larves, de même que la physiologie de la reine en relation avec son comportement de nidification.

Ainsi, le contróle de la pollinisation croisée efficace des plantes d'intérêt économique par les Bourdons ne sera pleinement assuré que lorsqu'un système pratique de domestication sera parfaitement adapté aux phénomènes de base de leur physiologie et de leur écologie.

Reçu pour publication en aqrit 1965.

\section{REMERCTEMENTS}

J'adresse de vifs remerciements à M. R. Robelin, Ingénieur divisionnaire S. P. V. P., Chef de la Division Sud, qui m'a autorisé à circuler dans toutes les parties du Domaine de Sceaux, me permettant de capturer les Insectes indispensables à mes travaux.

\section{SUMMARY}

A METIOD OF BUMBLE-BEES REARING;

FROM QUEENS CAPTURED IN THE FIELA)

Numerous investigators have pointed out that bumble-bees are more effective pollinators than honey-bees, where many important agricultural crops, particularly legumes, are concerned.

The method adopted by the author is based on catching bumble-bee queens in spring. The queens are released in a greenhouse where they fly about freely and where boxes have been set up in order to induce them to start nests. The Bumble-bees are fed with honey, a 50 per cent sugar solution, and crushed pollen. Jarge bourquets of flowers are placed in vases for the rueen's requirements of pollen.

The behaviour of the bumble-bee queens in the greenhouse is described, particularly feeding, the choice of nesting materials, the defence of the nest.

The results are shown in the table I.

\section{RÉFÉRENCES IBIBLIOGRAPHIQUES}

Free J. B., J3tetek C. (i., 195\%. Bumblebees. 'The New Naturalist. The Mac Millan Co; X. Y.

Fridex F., rg64. come swedish studies on bumblebees in captivity. Résumé de comnunication. Second Symposium international sur la Pollinisation. Londres.

Frison T. If., 1927. Experiments in rearing colonies of bumblebees (Bremidae) in artificial nests. Biol. Bull., 52, $5 \mathrm{I}-6 \%$.

Fye R. L., Mender I. T., 195ł. Field domiciles for bumblebees. J. econ. Entomol., 47, $672-676$.

Hasselrot T. B., 1952. A new method for starting bumblebee colonies. Agron. J., 44, 218-2 I9. Washington. 1960. Studies on swedish bunblebees (Genus Bombus LATr.) Opusc. Entomol. Suppl. XVII; Lund. $192 \mathrm{p}$.

Hoffer E., 1882. Die Hummeh Steiermarks. I. 92 p. Graz. 1883. Die Hummeln Steiermarks. II. 98 p. Graz.

Horber E., ig6r. Beitrag zur Domestikation der Ilummeln. Viertelj. Naturforsch. Gesellsch. Zürich, 106, $424-447$. 
Jorday R., 1936. Jïn Weg zur zwangsläufigen Nestgründung überwinterter Bombus-weibchen an einem bestimmten Platze. Arch. Bienenkde, 17, 39-44; Berlin-Zehlendorf.

LECOMTE J., 196r. Premier Symposium International sur la l'ollinisation (resumé). Copenhague (ig60). Ann. Abeille, 4, 67-70.

Lehmensick R., I956. Jiffolgreiche Versuche zur Hummelzucht in geschlossenen Raimen. Zool. Anz. 15\%, I 10-1 13. Leipzig.

LiNDHARd E., I9I2. Humblebien som Husdyr. Spredte Traek af nogle danske Humlebiarters Biologri. Tidskr. Landbrugets Plantecinl., 19, 335-352. Kobenharn.

Meder J. T., 1956. Principles and methods for the utilization of Bumblebees in cross-pollination of crops. Proc. Tenth Intern. Congr. Entomol., vol. 4.

Norgaard Holm S., 1960 . Experiments on the domestication of bumblebees (Bombus Latr.) in parti cular B. Lapidarius and B. terrestris. Roy. Veter. Agric. Coll. Copenhagen, Yearbook.

Pedersen A., 1945. Rödklöverens blomstring ogr bestövning. Den krol. Veter-og Landböhojskoles ársskrift. Plath O. E., 1923. Brecding experiments with confined Bremus (Bombus) queens. Biol. Bull. 45, 325-34r. Pouvreau A., r962. Contribution à l'étude de Sphaeruluria bomia (Nematoda, Tylenchida), parasite dés reines de Bourdons. Ann. Abeille, $5,181-199$

pouvreau A., rg64. Sur quelques ennemis des Bourdons. Communication, 2e Symposinm international sur la Pollinisation; Londres ( 1964$)$.

Sladen F. W. L., 1912. The Bumble-Bee. Mac Millan and Co; Jondon, $28,3 \mathrm{p}$.

STEIN (G., 1956. Beiträge zur Biologie der Ilummel (Bombus terrestris L., Bombus lapidarius L.). Zool. $J b .84,439^{-462}$

VALLE O., I955. Untersuchungen zur Sicherung der Bestiubung von Rotkleie. Acta 1gr. Fenn. 83, 205220. IIelsinki.

Zapletal F., I96I. Ueber die Domestikation der Hummeln. Arch.. Gen/gele. Kilrintierkile, 10. 